\title{
A Pericardial Cyst Causing Obstructive Shock
}

\author{
Mit Patel*, Injoon Lee, Neel Parikh, David Sane, Thomas Bishop
}

Virginia Tech Carilion School of Medicine, USA

\section{Article Info}

\section{Article Notes}

Received: June 17, 2019

Accepted: June 26, 2019

\section{*Correspondence:}

${ }^{*}$ Dr. Mit Patel, Virginia Tech Carilion School of Medicine, USA; Email: mvp5117@gmail.com.

(c) 2019 Patel M. This article is distributed under the terms of the Creative Commons Attribution 4.0 International License.

\section{Abstract}

Pericardial cysts are rare anomalies that are usually benign and asymptomatic. A prompt intervention is necessary when patients present with concerning clinical features. We report a case of pericardial cyst causing obstructive shock in a patient that underwent bowel resection after small bowel obstruction. Our patient underwent emergent cyst aspiration which alleviated the shock.

\section{Case Presentation}

A 71-year-old male was admitted for small bowel obstruction. CT of the abdomen showed a pericardial cyst of $10 \mathrm{~cm} \times 11 \mathrm{~cm}$ (Figure 1). A transthoracic echocardiogram was done to assess cardiac function (Figure 2). Upon further history with the patient, he was diagnosed with pericardial cyst $(<5 \mathrm{~cm})$ many years ago during a staging scan for his colon cancer, had followed with his PCP and oncologist, however, he was lost to follow up. Patient underwent lysis of adhesion, however after the surgery, the patient developed hypotension and lactic acidosis requiring vasopressors suggestive of shock. His post-op CT scan did not suggest of any bowel perforation or post-op complications. He underwent stat echocardiogram which showed signs of RV collapse. Interventional Radiology was consulted
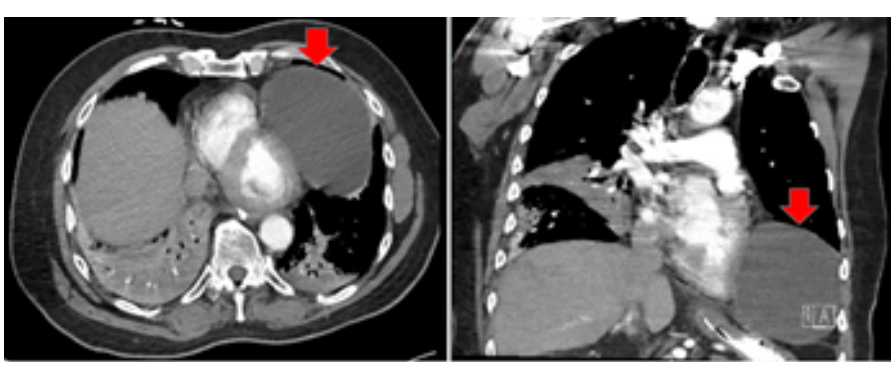

Figure 1: CT scan showing pericardial cyst on axial view (left) and coronal view (right).
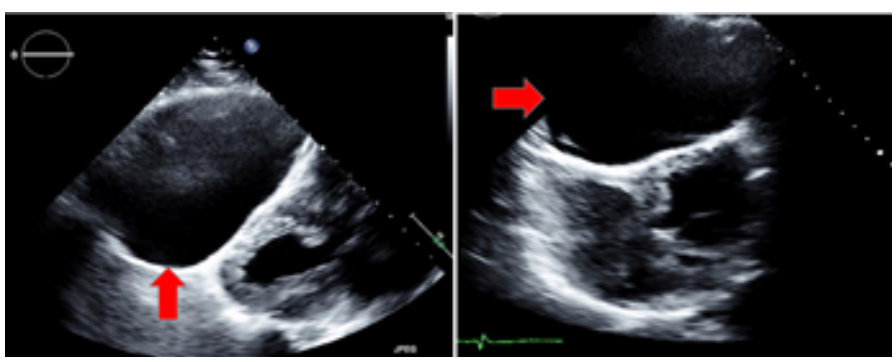

Figure 2: Echocardiogram showing diminished left ventricular size (left) and impaired right ventricular filling during diastole (right). 
for an emergent aspiration and 575cc of yellowish cloudy fluid was aspirated. Patient's lactic acidosis and shock resolved after the procedure. Fluid analysis was consistent with proteinaceous debris and rare benign cellular elements suggestive of histiocytes. Final diagnosis was cystic aspiration fluid.

\section{Conclusion}

Pericardial cysts typically remain asymptomatic. However, proper measures must be taken if symptoms occur especially if it is hemodynamically significant. Best imaging modality includes transthoracic echocardiogram, CT or MRI. Each modality offers its own distinct benefits over one another. Our patient presented with an obstructive shock which resolved with percutaneous drainage. Percutaneous drainage is an option, however $30 \%$ of the cases can recur, thus close follow up is necessary after the drainage. Video-assisted thoracoscopic surgery or robotic resections are permanent treatment options for removal. Each case must be discussed in a multi-disciplinary team between interventional cardiology, interventional radiology and cardiothoracic surgery to ensure the best possible outcome for the patient.

\section{Discussion}

Pericardial cysts are rare abnormalities that may cause life-threatening complications such as pericardial tamponade ${ }^{1}$. The most common locations for pericardial cysts include right cardiophrenic angle followed by left cardiophrenic angle and mediastinal locations not adjacent to the diaphragm ${ }^{2}$. Our patient's pericardial cyst was located overlying the apex of the RV and LV causing severe limitation to fill during diastole especially the RV. There was no collapse of the RV such as seen in cardiac tamponade, however, due to the mass effect, the right ventricle struggled to expand against substantial pressure. Thus, reduced forward from the RV and post-op hypovolemia combination led to significant lactic acidosis and hypotensive shock.

Pericardial cysts are mostly asymptomatic, however, when symptoms do appear, it presents as shoulder pain, chronic cough, chest pain, dyspnea, retrosternal pressure ${ }^{4}$. Compression of the adjacent structures are rare, however, if present, can be a life-threatening event requiring immediate intervention ${ }^{3,4}$. Pankaj Kaul et al. reported a 66-year-old woman presenting with congestive chest symptoms with features of right heart failure that was caused by a massive benign pericardial cyst ${ }^{5}$.
Most cases of pericardial cysts are diagnosed by routine chest x-ray, usually as an isolated cystic shadow adjacent to the heart. CT scan is considered the best imaging modality as it can provide delineation of the pericardial anatomy along with exact location and characterization of certain pericardial lesions which can help in surgical decision making. Disadvantages of CT scan include erroneous reporting if there is elevated protein content such as infection or hemorrhage, lack of functional assessment, radiation exposure, and need for breath holding. MRI provides excellent soft tissue architecture but is limited by poor calcification visualization and can be time consuming and expensive. Echocardiography is useful to assess the functional status of the heart but is not the preferred imaging modality due to narrow window of visualization which may increase the risk of missed pericardial cysts in unusual locations ${ }^{6}$.

Management of the cyst should follow a systematic approach based on the patient's symptoms, its urgency and potential complications associated with the procedure. It would be reasonable to follow asymptomatic patients with serial echocardiography or CT although CT would be less preferable due to its radiation exposure. Currently no clear cutoff in size exist, however if the size of the cyst is concerning for possible complication, then referral to cardiothoracic surgeon may be indicated. For symptomatic patients, percutaneous aspiration has been suggested as an acceptable treatment when surgery is high risk ${ }^{3,6}$. However, $30 \%$ of the patients experience recurrence, thus requiring VATS (video-assisted thoracoscopic surgery) or robotic resections as a permanent treatment ${ }^{3,6}$. Serial follow up is indicated after the procedure to ensure that the patient's symptoms are relieved, and no recurrence has ensued.

\section{References}

1. Patel J, Park C, Michaels J, et al. Pericardial cyst: case reports and a literature review. Echocardiography. 2004 Apr; 21(3): 269-72.

2. Stoller JK, Shaw C, Matthay RA. Enlarging, atypically located pericardial cyst. Recent experience and literature review. Chest. 1986 Mar; 89(3): 402-6.

3. Sharma R, Harden S, Peebles C, et al. Percutaneous aspiration of a pericardial cyst: an acceptable treatment for a rare disorder. Heart. 2007 Jan; 93(1): 22.

4. Sokouti M, Halimi M, Golzari S. Pericardial cyst presented as chronic cough: A rare case report. Tanaffos. 2012; 11(4): 60-62.

5. Kaul P, Javangula K, Farook S. Massive benign pericardial cyst presenting as simultaneous superior vena cava and middle lobe syndromes. Journal of Cardiothoracic Surgery. 2008 May; 3: 32.

6. Kar SK, Ganguly T. Current concepts of diagnosis and management of pericardial cysts. Indian Heart Journal. 2017 May; 69(3): 364-370. 